\title{
On the Possibility of Acceleration of Heavy Solar and Cosmic Nuclei To GeV, TeV Energies and Beyond
}

\author{
Shahinaz M. Yousef \\ Astronomy \& Meteorology Dept, Faculty of Science, Cairo University- Cairo Egypt \\ email: shahinazyousef@yahoo.com
}

\begin{abstract}
On acceleration of relativistic electrons by any means, the coherent pinch effect brings the relativistic electrons together. The inclusion of a suitable number of positive ions, preferentially heavy ions would stabilize the beam. The heavy trapped nuclei would have to go with the electrons, thus acquire relativistic speeds. The electron beam may be divided into small bunches. This mechanism can accelerate positive nuclei to $\mathrm{GeV}$ and $\mathrm{TeV}$ energies during solar flares, stellar and galactic high energy events. During the acceleration of $1000 \mathrm{GeV}$ electron bunches, the energy gained by trapped positive nuclei is estimated to reach $10^{15} \mathrm{eV}$ per nucleon. Higher energies can be achieved by head on collision as the energy gained by the target nucleus is proportional to $\gamma^{2}$, however fission reactions and very high energy gamma rays may occur. The principle of this mechanism is applied in electron-ring accelerators.
\end{abstract}

Keywords. Sun: particle emission

\section{Introduction}

Energetic particles from impulsive flares show that elements with $\mathrm{Z}>8$ are strongly enhanced relative to coronal abundance. Elements up to Si are fully ionized and Fe has charge 20. There is a strong evidence of electron beams in these events (Reames 1996). The observed number of relativistic heavy solar and cosmic ray nuclei (Schatzman 1967) can only be explained as due to an acceleration mechanism which:- 1) Preferentially accelerates heavy nuclei. 2) Simultaneously accelerates electrons and nuclei. 3) Accelerates all particles to the same velocity.

\section{Coherent acceleration mechanism}

At relativistic velocities, coherent effect occurs in intense electron beams in which the electrons themselves generate appreciable magnetic field (the pinch effect). The magnetic attraction force $F_{m}$ is related to the repulsive electric force $F_{e}$ by the relation, Kerst (1966)

$$
F_{m}=\left(\frac{V^{2}}{c}\right) F_{e}
$$

At velocities $V \sim c$, a pure beam of electrons would diverge slowly but could be rendered stable by the seeding of a suitable number of positive charges. These positive ions would be trapped electrostatically in the beam's very deep potential well and would necessarily acquire the same velocity of the electrons on acceleration. The ions are actually accelerated by the internal coherent electric field of the electrons (Veksler et al. 1967). 
For a perfect coherent mechanism, the radius of the beam $L$ should be $L \leqslant \lambda$ where $\lambda$ is the plasma wavelength. For the bunch to be coherent in all directions, the height of the cylinder should equal $2 L$.

If $V$ is the velocity of the bunch and $\omega_{0}$ is the plasma frequency, then

$$
\lambda=\frac{V}{\omega_{0}}, \quad \text { where } \omega_{0}^{2}=\frac{4 \pi e^{2} N_{e}}{m}
$$

$m$ and $e$ are the mass and charge of the electron and $N_{e}$ is the electron density. Assuming $V=0.9 c, N_{e}=10^{12} \mathrm{~cm}^{-3}$ then $\lambda=0.48 \mathrm{~cm}$ and if $N_{e}=10^{11} \mathrm{~cm}^{-3}$ then $\lambda=1.5 \mathrm{~cm}$.

\section{Head on collisions}

Consider the collision of a fast relativistic particle of mass $M_{1}$ with an immobile particle of mass $M_{2}$, where $M_{2} \ll M_{1}$. If $M_{1} \gg M_{2} \gamma$ then as a result of such head on collision, the immobile particle will get the following energy

$$
W=M_{2} c^{2} \gamma^{2} \text { Where } \gamma=\left[1-\left[\frac{V}{c}\right]^{2}\right]^{\frac{-1}{2}}
$$

Veksler (1956) assumed the primary relativistic particle of Mass $M_{1}$ to consist of $n_{1}$ electrons and the immobile mass $M_{2}$ to consist of $n_{2}$ particles of mass $m_{2}$, then if the condition

$$
n_{1} m_{1} \gg n_{2} m_{2} \gamma
$$

is fulfilled, then every ion of mass $m_{2}$ would obtain energy

$$
W=m_{2} c^{2} \gamma^{2}
$$

This mechanism can give very high energies to cosmic ray particles as the energy gained by each particle is proportional to $\gamma^{2}\left(\gamma^{2}\right.$ increases from 1.33 for $V / c=0.5$ to 500.5 for $V / c=0.999)$. This mechanism is capable of producing energies in the $\mathrm{TeV}$ and ultra relativistic range, however, the possibility of fission of heavy nuclei may exists. A target proton of rest mass $938 \mathrm{MeV}$, on moving with almost the velocity of light after the head on collision can acquire energies as large as $468.995 \mathrm{GeV}$. An iron nucleus moving with the same speed would acquire more than $26 \mathrm{TeV}$.

\section{Conclusions}

The steps of the proposed mechanism for the preferential acceleration of heavy solar and cosmic nuclei are:

1. The formation and acceleration of high density electron beams of radius $L \leqslant \lambda$, where $\lambda$ is the plasma wavelength. Pinching will increase the density of the beam. Instabilities in the electron beam, might break it into small bunches.

2. On seeding the bunches with ions, they will be stripped of their electrons. Heavy ions get ionized more effectively than low $Z$ ones and due to electrostatic attraction, the electron bunches preferentially collects high $Z$ (Levy 1968).

3. The acceleration of the self focused positively seeded bunches of electrons as a whole, thus the ions are forced to acquire the same velocity of the electrons.

4. Since $1000 \mathrm{GeV}$ electrons have been detected in cosmic rays, then the energy gained by a trapped nuclei can be $1.8 \times 10^{15} \mathrm{eV}$ per nucleon. 
5. On head on collision, the stabilized electron bunch can give energy to target nuclei that is proportional to $\gamma^{2}$. $\Lambda 1000 \mathrm{GeV}$ electron bunch would convey ultra relativistic energies to a target nucleus and might cause fission.

6 . The solar millisecond radio spikes require a coherent radiation mechanism that is also needed to explain some radio bursts on the sun and other active stars (Lang, 2001). Such spikes may be the site for coherent electron acceleration.

The general principle of this mechanism is applied in electron ring accelerators (Veksler et al. 1967 and Keefe 1969).

\section{References}

Kerst, D.W. 1966, The Development Of High energy Accelerators, Classics of Science (edited By M.S. Livingstone) VIII, Dover, New York, 1966.

Keefe, D. 1969, Science J., 5, 71 (1969).

Lang, K.R 2001, The Cambridge Encyclopedia of the Sun. Cambridge University Press.

Levy, R. H., in Symposium on Electron Ring Accelerators, Lawrence Radiation Laboratory Report UCRL 18103, 318 (1968).

Reames D.V., Energetic Particles from Solar Flares and Coronal Mass Ejections (1996). http://lheawww.gsfc.NASA.gov/Reames/cv.html

Schatzman, E., "High Energy Astrophysics" Lecture delivered at Les Houches during the 1966 Summer School of Theoretical Physics) edited by C. Dewitt, E. Schatzman and P. Vernon, Gordon and Breach, 1967.

Veksler, V.I., in proceedings of the CERN. symposium on High Energy Accelerators and Pion Physics, Geneva, Switzerland 1, 68, 1956.

Veksler, V.I., Sarantsev, V.P., Bonch-Osmolovsky, A.G., Dolbilov, G.V., Ivanov, G.A., Ionovitch, M.L., Kozhukhov, I,V., Kusnetsev, S.B., Makhan'kov, V.G., Perel'shtein, E.A., Rashevsky, V.P., Reshetnikova, K.A. Rubin, N.B., Ryl'stev, P.I., and Yarkovey, O.I., Proceedings of the sixth international conference on accelerators, Cambridge, Mass., U.S.A, 1967. 